\title{
On the Minimum Node Degree and Connectivity of a Wireless Multihop Network
}

\author{
Christian Bettstetter ${ }^{*}$ \\ Technische Universität München (TUM) \\ Institute of Communication Networks \\ D-80290 Munich, Germany \\ http://www.lkn.ei.tum.de \\ Christian.Bettstetter@ei.tum.de
}

\begin{abstract}
This paper investigates two fundamental characteristics of a wireless multihop network: its minimum node degree and its $k$-connectivity. Both topology attributes depend on the spatial distribution of the nodes and their transmission range. Using typical modeling assumptions - a random uniform distribution of the nodes and a simple link model — we derive an analytical expression that enables the determination of the required range $r_{0}$ that creates, for a given node density $\rho$, an almost surely $k$-connected network. Equivalently, if the maximum $r_{0}$ of the nodes is given, we can find out how many nodes are needed to cover a certain area with a $k$-connected network. We also investigate these questions by various simulations and thereby verify our analytical expressions. Finally, the impact of mobility is discussed.

The results of this paper are of practical value for researchers in this area, e.g., if they set the parameters in a network-level simulation of a mobile ad hoc network or if they design a wireless sensor network.
\end{abstract}

\section{Categories and Subject Descriptors}

C.2 [Computer-communication networks]: Network architecture and design-wireless communication, network communications, network topology; G.2.2 [Discrete mathematics]: Graph theory; F.2.2 [Probability and statistics]: Stochastic processes

\section{General Terms}

Design, Performance, Theory

\footnotetext{
${ }^{*}$ This work is funded by the German Research Foundation DFG within the program AKOM (Adaptability in heterogenous communication networks with wireless access).
}

Permission to make digital or hard copies of all or part of this work for personal or classroom use is granted without fee provided that copies are not made or distributed for profit or commercial advantage and that copies bear this notice and the full citation on the first page. To copy otherwise, to republish, to post on servers or to redistribute to lists, requires prior specific permission and/or a fee.

MOBIHOC'02, June 9-11, 2002, EPF Lausanne, Switzerland.

Copyright 2002 ACM 1-58113-501-7/02/0006 ...\$5.00.

\section{Keywords}

Ad hoc networking, sensor networks; connectivity, minimum node degree, isolated nodes; analytical methods, modeling, geometric random graphs.

\section{INTRODUCTION}

Wireless multihop networks are formed by a group of nodes that communicate with each other over a wireless channel. They operate in a decentralized and self-organizing manner and do not rely on fixed network infrastructure. Each node can act as a router to forward traffic toward its destination.

While the fundamental idea of such packet radio networks goes back to at least the 1970s [1], recent years have shown a tremendous comeback of research in this area (see, e.g., [2, $3,4]$ ). A great advantage of mobile wireless multihop systems is that they can be formed in a spontaneous and fast way; this is why they are called "ad hoc networks." Several application scenarios, such as ad hoc communication between mobile computers for conferencing and home networking, wireless sensor networks [5], multihop extensions of cellular telecommunication systems [6], and networks of vehicles $[7,8]$ are in the minds of researchers and developers.

Good progress has been made in the development of protocols (e.g., routing, medium access) that take the unique characteristics of ad hoc networks into account, but less work has been done to investigate ad hoc networks in an analytical manner and to find a convenient and exact mathematical description for modeling. In this paper, we address the latter two issues.

We investigate a very fundamental and important property of wireless multihop networks, namely their connectivity. Whereas in wireless networks with fixed infrastructure (e.g., cellular telecommunication networks or wireless LANs), it is sufficient that each mobile node has a wireless link to at least one base station, the situation in a decentralized ad hoc network is more complicated. To achieve a fully connected ad hoc network, there must be a wireless multihop path from each mobile node to each other mobile node. The connectivity therefore depends on the number of nodes per unit area (node density) and their radio transmission range. Each single mobile node contributes to the connectivity of the entire network.

The correct adjustment of the nodes' radio transmission 
power is therefore an important system feature. As in cellular networks, power adjustment can reduce interference while maintaining a certain Quality of Service. In ad hoc networks, it also allows the controlling of the topology of the network $[9,10]$. If we increase the transmission power of a node, it will typically achieve a higher transmission range and therefore reach more other nodes via a direct link. On the other hand, if we make the transmission power of a node very low, the node may become isolated without any link to other nodes.

In this context, this paper addresses the following questions: For a given number of nodes $n$ per area $A$, what is the minimum radio transmission range $r_{0}$ required to achieve

- an ad hoc network in which no node is isolated?

- a fully connected ad hoc network, i.e., a network in which each node can reach each other node via a wireless multihop path?

This range assignment problem arises, for example, if we want to set the system parameters for network-level simulations of ad hoc networks. We investigate this question analytically and by simulation under typical modeling assumptions, namely a random uniform node distribution with $n$ nodes on a system area $A$ and a simple channel model in which each node has the same transmission range $r_{0}$.

An equivalent problem must be solved in the system design of wireless multihop networks. For example, a largescale sensor network should cover a certain area $A$ to perform environmental monitoring. The used sensor type can transmit a range $r_{0}$ in the given environment (e.g., free space). How many sensors of this type do we need to obtain a connected network (see also [11])?

In addition to the basic problems "no isolated node" and "connected network," we also consider a network design that is robust against node and link outages. That is, how can we achieve with minimized resources

- an ad hoc network in which each node has at least $n_{0}$ neighbors $\left(n_{0} \geq 1\right)$ ?

- an ad hoc network that will still be connected if any $k-1$ nodes fail ( $k$-connected network)?

Before we present the solutions to these problems, Section 2 describes the used network model in more detail, and Section 3 recalls some basic definitions of graph theory and introduces the terms used in this paper. The main contributions of this paper are presented in Sections 4 and 5 .

In Section 4, we derive an expression for the probability that each node in the network has at least $n_{0}$ neighbors $\left(n_{0}=0,1,2 \ldots\right)$. For given parameters $(n, \rho)$ we can state how to set the range $r_{0}$ to achieve, with a certain probability $p$, a network with no isolated node. We also investigate these questions by simulations and thereby verify our analytical expressions. To solve the problem of a connected network (Section 5), we model an ad hoc network as a so-called geometric random graph. A recently published theorem in this mathematical research field allows us to make some interesting statements about the probability that a homogeneous ad hoc network is connected or even $k$-connected. Most important, we can calculate the range $r_{0}$ and density $\rho$ that is required to obtain an almost surely $k$-connected network. Again, we perform simulations for $k=1,2$, and 3 .

Section 6 discusses the impact of mobility on our results, and Section 7 outlines related articles and compares them to our contributions. Finally, Section 8 concludes this paper.

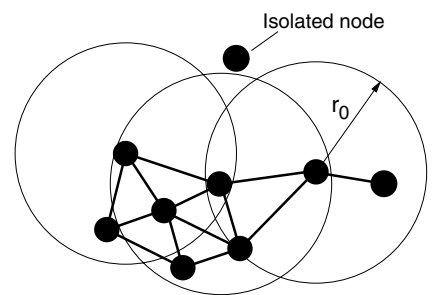

Figure 1: Modeling the topology of ad hoc networks

\section{NETWORK MODEL}

Three fundamental models are needed to represent the dynamic topology of a wireless ad hoc network in system-level investigations: (a) a model for the spatial node distribution, (b) a model for the wireless channel between the nodes, and (c) a model for the movement behavior of the nodes. A common way to do this is as follows:

From a set of $n$ network nodes, each node is independently randomly placed on a two-dimensional simulation area $A$. A uniform random distribution is used, such that for large $n$ and large $A$ we can define a constant node density $\rho=n / A$. It denotes the expected number of nodes per unit area.

To model the wireless transmission between the nodes, a radio link model is assumed in which each node has a certain transmission range $r_{0}$ and uses omnidirectional antennas. As illustrated in Figure 1, two nodes are able to communicate directly via a wireless link, if they are within range of each other. Only bidirectional links are considered. This link model corresponds to a propagation model with a certain signal attenuation (path loss). Let $P_{0}=P(r=0)$ denote the transmitted signal power at the sending node and $P(r)$ the received power at a distance $r$ from the sender. The received power falls off as $P(r) \propto r^{-\gamma} P_{0}$, where $\gamma$ is the path loss exponent, which depends on the environment (typically $2 \leq \gamma \leq 5)$. The wireless transmission range $r_{0}$ can then be mapped to the equivalent transmission power $P_{0}$ using a threshold for receiver sensitivity $P_{s}$. A node can receive properly if $P\left(r=r_{0}\right) \geq P_{s}$.

All nodes are free to move in the system area according to a certain mobility model (e.g., random waypoint, random direction, street model, etc.). This issue will be discussed in Section 6.

In summary, such a network model is not very sophisticated, but it is well-suited for investigations of the fundamental properties of the network topology (such as connectivity) and the behavior of distributed algorithms (e.g., routing, leader election, radio resource management, topology control).

\section{SOME BASIC GRAPH THEORY}

With this network model, we can represent an ad hoc network at each time instant as an undirected graph $G$. A graph $G=G(V, E)$ consists of a set of $n$ nodes (vertices) and a set of $m$ node pairs (edges, links). The set of nodes, denoted by $V=\{1, \ldots, n\}$, represent the network-enabled ad hoc devices; and the set of edges, denoted by $E$, represent the wireless communication links. Since our channel model only considers bidirectional communication links and ignores unidirectional links, we only consider undirected graphs, i.e., all link relations on node pairs are symmetric. In order to understand the following sections, we recall some basic def- 


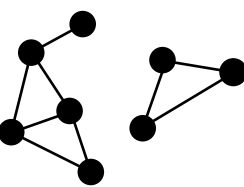

a. unconnected $G$

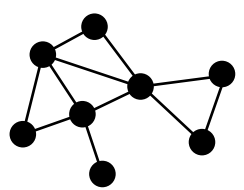

b. (1-)connected $G$

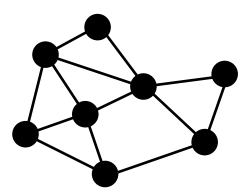

c. 2-connected $G$
Figure 2: Illustration of graph connectivity

initions of graph theory and define the nomenclature used in this paper.

\subsection{Node Degree}

The degree of a node $u$, denoted as $d(u)$, is the number of neighbors of node $u$, i.e., its number of links. A node of degree $d=0$ is isolated, i.e., it has no neighbors (see Fig. 1). The minimum node degree of a graph $G$ is denoted as

$$
d_{\min }(G)=\min _{\forall u \in G}\{d(u)\} \text {. }
$$

The average (or mean) node degree of $G$ is

$$
d_{\text {mean }}(G)=\frac{1}{n} \sum_{u=1}^{n} d(u) .
$$

In an undirected graph we have $d_{\text {mean }}(G)=2 m / n$ [12] .

\subsection{Graph Connectivity}

As illustrated in Fig. 2, a graph is said to be connected, if for every pair of nodes there exists a path between them, and otherwise it is disconnected [13]. In terms of communication networks, all nodes of a connected network can communicate with each other over one or multiple hops (links), whereas in a disconnected network we have several islands of subnetworks, whose nodes form a connected subgraph but cannot reach other subnetworks.

Moreover, a graph is said to be $k$-connected ( $k=$ $1,2,3, \ldots)$ if for each node pair there exist at least $k$ mutually independent paths connecting them (see Fig. 2c, $k=2$ ). Equivalently, a graph is $k$-connected if and only if no set of $(k-1)$ nodes exists whose removal would disconnect the graph [13]. In other words, if $(k-1)$ nodes fail, the graph is guaranteed to be still connected. The maximum value of $k$ for which a connected graph is $k$-connected is the connectivity $\kappa$ of $G$ [13]. It is the smallest number of nodes whose failure would disconnect $G$.

Similarly, a graph is called $k$-edge-connected if and only if there are at least $k$ edge-disjoint paths between every pair of nodes. If a graph is $k$-connected, then it is also $k-$ edge-connected, but the reverse implication is not necessarily true. The edge connectivity $\lambda(G)$ is defined analogously to the (node) connectivity $\kappa(G)$.

For every graph with at least two nodes, we have $\kappa(G) \leq$ $\lambda(G) \leq d_{\min }(G)[13]$.

\section{ISOLATED NODES AND MINIMUM DEGREE}

\subsection{Isolated Nodes}

The existence of isolated nodes is certainly an undesirable characteristic of a wireless multihop network. In a non-mobile wireless multihop network, e.g., a static sensor network, an isolated node cannot exchange any information with other nodes and is therefore useless for the entire community of nodes. In a mobile scenario, an isolated node that wants to send or receive information must wait until it moves into the range of another node or until another node passes by. This might cause an unacceptable message delivery delay.

Let us therefore consider the following range assignment problem: A set of $n$ nodes, each node with a transmission range $r_{0}$, are randomly uniformly placed in a large area $A \gg$ $r_{0}^{2} \pi$ (node density $\rho=n / A$ ). What is the minimum range $r_{0}$ such that, with high probability $p$, no node in the network is isolated (i.e., each node has at least one neighbor).

To solve this problem, we employ nearest neighbor methods known from analysis of spatial data (see, e.g., [14]). Such methods are used in many sciences to analyze certain point patterns, for example, in ecology, forestry, and cosmology. The distance of a point to its closest neighboring point is denoted as its nearest neighbor distance $\xi$. For a homogeneous Poisson point process in two dimensions (constant intensity $\rho)$, the probability density function of the nearest neighbor distance is (see [14], Chapter 8)

$$
f(\xi)=2 \pi \rho \xi \cdot e^{-\rho \pi \xi^{2}} \text { for } \xi>0
$$

with mean value (see Appendix A)

$$
E(\xi)=\int_{0}^{\infty} \xi p(\xi) d \xi=\frac{1}{2 \sqrt{\rho}} .
$$

In our application, a "random point" represents a "random node" of the ad hoc network. Thus, the probability that the distance between a randomly chosen node to its nearest neighboring node is less than or equal $r$ is

$$
P(\xi \leq r)=\int_{\xi=0}^{r} f(\xi) d \xi=1-e^{-\rho \pi r^{2}} .
$$

If we set the range of all nodes to $r=r_{0}$, Equation (5) denotes the probability that a node $u$ has at least one neighbor, i.e.,

$$
P(d(u)>0)=P\left(\xi \leq r_{0}\right) .
$$

The probability that a node has no neighbor (i.e., it is isolated) is therefore

$$
\begin{aligned}
P(d(u)=0) & =P\left(\xi>r_{0}\right)=1-P\left(\xi \leq r_{0}\right) \\
& =e^{-\rho \pi r_{0}^{2}} .
\end{aligned}
$$

Our aim is to achieve a network graph $G$ in which none of the $n$ nodes is isolated, i.e., $d(u)>0, \forall u \in G \Leftrightarrow d_{\min }(G)>$ 0 . Assuming statistical independence, the probability for this event is

$$
\begin{aligned}
P\left(d_{\min }>0\right) & =\left(\begin{array}{l}
n \\
n
\end{array}\right) P(d>0)^{n} P(d=0)^{0} \\
& =\left(1-e^{-\rho \pi r_{0}^{2}}\right)^{n} .
\end{aligned}
$$

Figure 3 shows two example plots of this function over $r_{0}$ and $n$ for $A=10^{6} \mathrm{~m}^{2}$ and $A=4 \cdot 10^{6} \mathrm{~m}^{2}$. We conclude this section with the following theorem.

Theorem 1 (A probabilistic bound to avoid isolated nodes in homogeneous ad hoc networks): Given is an ad hoc network with $n \gg 1$ nodes and a homogeneous node density $\rho$ in nodes per unit area. If we want to be sure, with a probability of at least $p$, that no node in this ad hoc network 


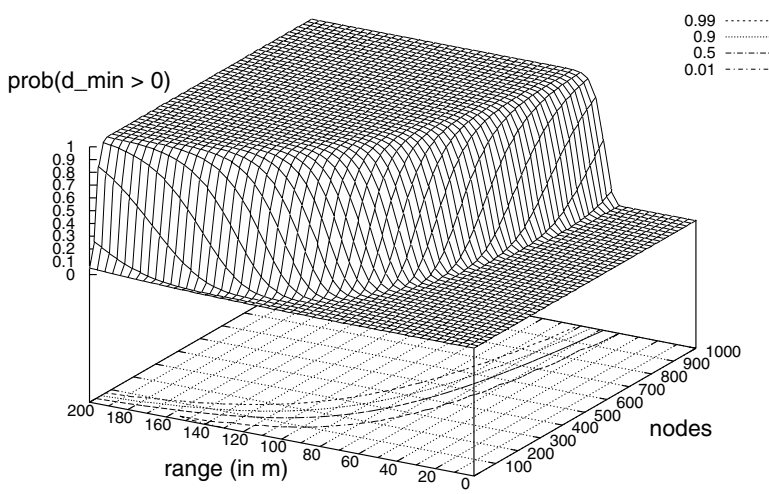

a. System area $A=10^{6} \mathrm{~m}^{2}$ (e.g., $1000 \times 1000 \mathrm{~m}^{2}$ )

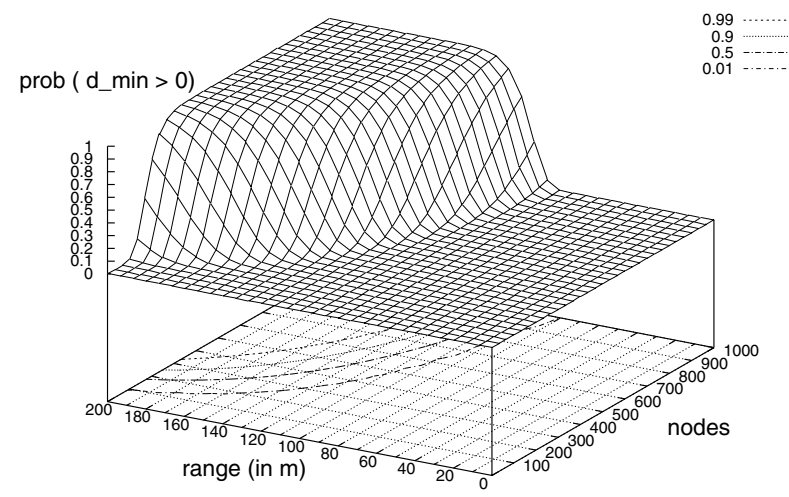

b. System area $A=4 \cdot 10^{6} \mathrm{~m}^{2}$ (e.g., $2000 \times 2000 \mathrm{~m}^{2}$ )

Figure 3: Probability that no node is isolated $P\left(d_{\min }>0\right)$

is isolated, i.e., $d_{\min } \geq 1$, we can set the radio range of all nodes to

$$
r_{0} \geq \sqrt{\frac{-\ln \left(1-p^{1 / n}\right)}{\rho \pi}} .
$$

The following example illustrates the usefulness of this equation.

Example 1 (Simulation of an ad hoc network): Image that we perform a simulation-based study of an ad hoc network; for example, we investigate the behavior of a distributed leader election algorithm. A uniform random generator is used to position $n=500$ nodes in a simulation area of size $A=1000 \times 1000 \mathrm{~m}^{2}$, which yields a node density of $\rho=$ $5 \cdot 10^{-4} \mathrm{~m}^{-2}$. We would like to achieve that almost surely no node in the network is isolated. To do so, we require a probability of at least $p=99 \%$. According to (9), we can set the transmission range of all nodes to $r_{0}=83 \mathrm{~m}$ or higher to achieve this goal.

If our nodes are only capable of transmitting $r_{0}=70 \mathrm{~m}$, we will need at least $n=728$ nodes to achieve the same confidentiality (also see Fig. 3a). In a scenario with $n=100$ nodes in the same area, each node must cover a range of $r_{0} \geq 172 \mathrm{~m}$. Certainly, a higher range and/or more nodes will be required if we use a larger area (Fig. 3b).

\subsection{Minimum Node Degree}

Until now we required that each node has at least one neighbor. A generalization of this problem is to require that each node has at least a certain number, say $n_{0}$, neighbors. In other words, the resulting network graph should have a certain minimum degree $d_{\min }(G) \geq n_{0} \Leftrightarrow \min _{\forall u \in G} d(u) \geq$ $n_{0}$. A high node degree makes an ad hoc node more resistant against failures of neighbors and links. Furthermore, in some classes of ad hoc networks, there is an optimal number of neighbors that each node should have, e.g., to achieve an optimal throughput [15].

In the following, we derive a probabilistic expression that allows us to calculate the critical radio range that is required to achieve a certain $d_{\min }(G)$ for given $n$. Equivalently, we can calculate the critical number of nodes $n$ for given $r_{0}$.

We first solve this problem on a one-dimensional line, and then generalize the result for the two-dimensional case. Let the random variable $d$ denote the number of neighbors of a node.

\subsubsection{One-dimensional case}

We consider the following homogeneous Poisson point problem in one dimension (compare with [16], Section 3.4): A number of $n$ nodes are randomly uniformly positioned in an interval $\left[0, x_{\max }\right]$. What is the probability that $n_{0}$ of these $n$ nodes are located in the interval $\left[x_{1}, x_{2}\right]$, where $0 \leq x_{1} \leq x_{2} \leq x_{\max }$ ? The random variable $d^{*}$ denotes the number of nodes within the given interval.

The probability that a node is placed within the interval $\left[x_{1}, x_{2}\right]$ is $p=\frac{x_{2}-x_{1}}{x_{\max }}$. The probability that $n_{0}$ of $n$ nodes are placed in the interval $\left[x_{1}, x_{2}\right]$ is

$$
P\left(d^{*}=n_{0}\right)=\left(\begin{array}{c}
n \\
n_{0}
\end{array}\right) p^{n_{0}}(1-p)^{n-n_{0}} .
$$

For $n \gg 1$ and $\left(x_{2}-x_{1}\right) \ll x_{\max }$, we can approximate this solution with a Poisson distribution, i.e.,

$$
P\left(d^{*}=n_{0}\right)=\frac{(n p)^{n_{0}}}{n_{0} !} \cdot e^{-n p}
$$

for $n_{0}$ in the order of $\frac{x_{2}-x_{1}}{x_{\max }} n$.

Let us now suppose large $n$ and large $x_{\max }$ but keep the ratio $n / x_{\max }$ constant. The density $\rho=n / x_{\max }$ is then the expected number of nodes per unit length. For given density $\rho$ we can calculate the probability that $n_{0}$ nodes are in an interval of length $x_{0}=x_{2}-x_{1}$ as

$$
P\left(d^{*}=n_{0}\right)=\frac{\left(\rho x_{0}\right)^{n_{0}}}{n_{0} !} \cdot e^{-\rho x_{0}} .
$$

The interval $\left[x_{1}, x_{2}\right]$ could be a symmetric interval around a randomly chosen position $x_{p}=\frac{x_{0}}{2}$ on the line, i.e., $\left[x_{1}, x_{2}\right]=\left[x_{p}-\frac{x_{0}}{2}, x_{p}+\frac{x_{0}}{2}\right]$. In general, $x_{p}$ does not necessarily represent the position of a node on the line. However, for large $n$, if we choose one node, the other $(n-1)$ nodes are still uniformly distributed on the line. Thus, Equation (12) also denotes the probability that a node has $n_{0}$ neighbors if the radio range is set to $r_{0}=x_{0} / 2$ :

$$
P\left(d=n_{0}\right)=\frac{\left(\rho 2 r_{0}\right)^{n_{0}}}{n_{0} !} \cdot e^{-\rho 2 r_{0}} .
$$

The probability that a node is isolated, i.e., it has no neighbors is $P(d=0)=e^{-\rho 2 r_{0}}$. 


\subsubsection{Two-dimensional Case}

The same problem in two dimensions is: what is the probability that $n_{0}$ of all $n$ nodes are within a certain area $A_{0}$ in the system plane $A$ ? The entire system area $A$ is for example a large rectangular area or a disc.

To solve this problem, we can replace the system interval $\left[0, x_{\max }\right]$ by the system area $A$ and the subinterval $x_{0}$ by a subarea $A_{0}$. The expected number of nodes per unit area is then $\rho=\frac{n}{A}$.

Analogous to (12), the probability of finding $n_{0}$ nodes in an area $A_{0}$ is thus

$$
P\left(d^{*}=n_{0}\right)=\frac{\left(\frac{A_{0}}{A} n\right)^{n_{0}}}{n_{0} !} \cdot e^{-\frac{A_{0}}{A} n}=\frac{\left(\rho A_{0}\right)^{n_{0}}}{n_{0} !} \cdot e^{-\rho A_{0}}
$$

for large $n$ and large $A$. A radio range $r_{0}$ covers an area $A_{0}=\pi r_{0}^{2}$. Thus, the probability that a randomly chosen node has $n_{0}$ neighbors is

$$
P\left(d=n_{0}\right)=\frac{\left(\rho \pi r_{0}^{2}\right)^{n_{0}}}{n_{0} !} \cdot e^{-\rho \pi r_{0}^{2}}
$$

It is isolated with a probability of $P(d=0)=e^{-\rho \pi r_{0}^{2}}$ (see (7)). The expected number of neighbors of a node is

$$
E(d)=\rho \pi r_{0}^{2},
$$

which is the average node degree $d_{\text {mean }}$ of the resulting graph. (It can also be calculated by $E(d)=$ $\int_{r=0}^{r_{0}} \int_{\phi=0}^{2 \pi} \rho(r, \phi) r d r d \phi$.) Again, we consider all nodes of the network and conclude with the following theorem.

Theorem 2 (A probabilistic bound for the minimum node degree of a homogeneous ad hoc network): Given is an ad hoc network with $n \gg 1$ nodes, each with range $r_{0}$, and a homogeneous node density $\rho$ in nodes per unit area. The probability that each node has at least $n_{0}$ neighbors, i.e., the network has a minimum node degree $d_{\min } \geq n_{0}$, is given by

$$
P\left(d_{\text {min }} \geq n_{0}\right)=\left(1-\sum_{N=0}^{n_{0}-1} \frac{\left(\rho \pi r_{0}^{2}\right)^{N}}{N !} \cdot e^{-\rho \pi r_{0}^{2}}\right)^{n}
$$

Figure 4 shows $P\left(d_{\min } \geq 2\right)$ and $P\left(d_{\min } \geq 3\right)$ over $r_{0}$ and $n$ for an area of size $10^{6} \mathrm{~m}^{2}$. The plot of $P\left(d_{\text {min }} \geq 1\right)$ has been shown in Figure 3a.

Example 1 (continued): We continue the previous example with $n=500$ nodes on $A=10^{6} \mathrm{~m}^{2}$. In order to achieve an ad hoc network in which each node has almost surely $(p=99 \%)$ at least two neighbors, a transmission range of $r_{0}>93 \mathrm{~m}$ is required (Fig. 4a); a range $r_{0}>100 \mathrm{~m}$ is needed to achieve three neighbors (Fig. $4 \mathrm{~b}$ ). In the latter case, the average number of neighbors of a node is $d_{\text {mean }}=15.7$. If the maximum transmission power allows the nodes to transmit only $r_{0}=70 \mathrm{~m}$, a set of $n=920$ nodes must be used to achieve $P\left(d_{\text {min }} \geq 2\right)>99 \%$.

\subsection{Simulations and Discussion}

Let us now verify these analytical results by computer simulation. In our simulation environment, a uniform random generator [17] chooses the $x$ and $y$ coordinates of $n=500$ nodes on a $1000 \times 1000 \mathrm{~m}^{2}$ system area. For a given radio range $r_{0}$, the links between the nodes are created, and the minimum node degree $d_{\min }$ of the resulting network topology is determined. This experiment is repeated 10000

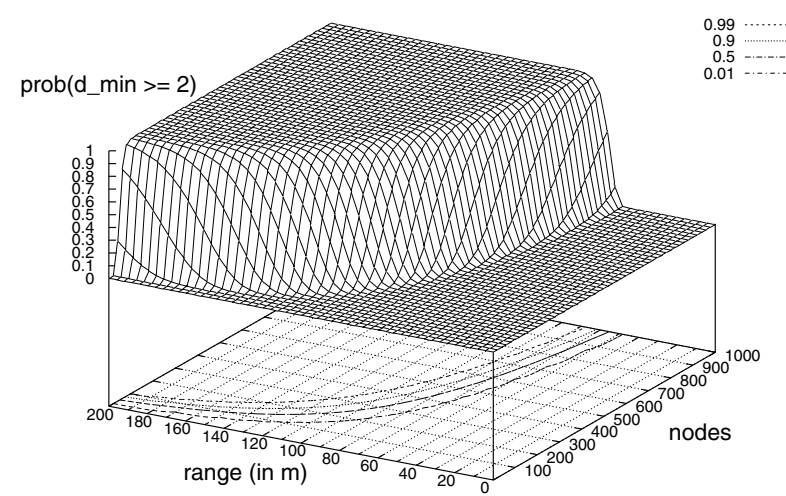

a. $P\left(d_{\min } \geq 2\right)$ : Prob. that each node has at least 2 neighbors

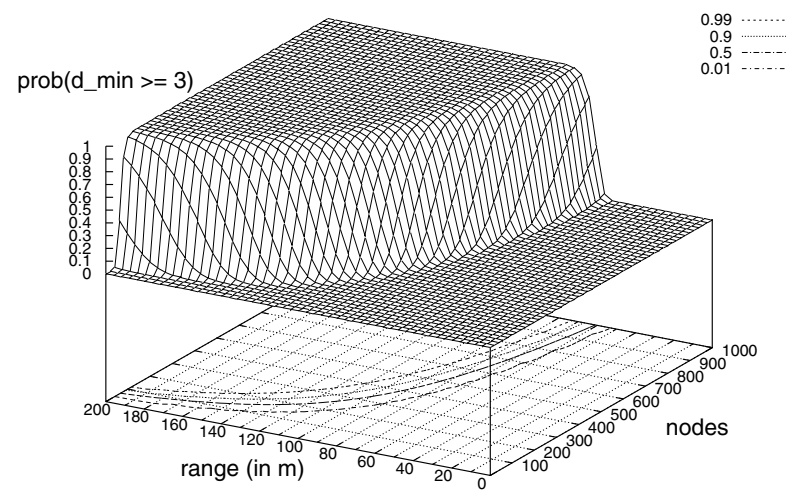

b. $P\left(d_{\min } \geq 3\right)$ : Prob. that each node has at least 3 neighbors

Figure 4: Probability that each node has at least $n_{0}$ neighbors, $P\left(d_{\min } \geq n_{0}\right)$, in a system area $A=10^{6} \mathbf{m}^{2}$ (e.g., $1000 \times 1000 \mathbf{m}^{2}$ ): Analytical curve over range $r_{0}$ and nodes $n_{0}$

times, and finally averaged over all 10000 random topologies, which yields an acceptable confidentiality of the obtained results. The resulting curves are shown in Figure 5a.

We observe that this simulation yields the same qualitative behavior as the analytical plots shown in Figs. 3 and 4 - however, there is a significant quantitative difference. In the simulation, a much higher transmission range is required to achieve the same $P\left(d_{\min }\right)$. For example, about $r_{0}=105 \mathrm{~m}$ instead of $83 \mathrm{~m}$ is needed to create a network with no isolated node.

Are our formulas wrong? No, they are not. The difference results from the fact that the simulation was done on a bounded area, whereas our analytical derivation assumed an infinite large area. In the simulation environment, nodes located at the edges and borders of the area can only have links toward the middle of the area. Thus, their node degree is on average lower than that of nodes in the middle. This border effect makes it impossible to compare the results of the simulation with the analytical formulas. In fact, the analytical value for $r_{0}(p)$ (Theorem 1 ) is a lower bound for the range that is required in a simulation environment, and $P\left(d_{\min } \geq n_{0}\right)$ of Theorem 2 is an upper bound for the probability that each node in the simulation has at least $n_{0}$ neighbors.

But there are methods to avoid such a border effect [14]. A 


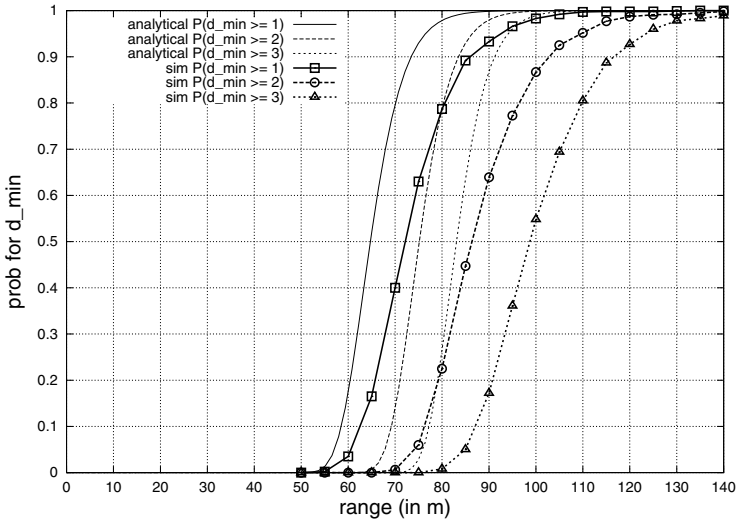

a. $P\left(d_{\min } \geq n_{0}\right)$ with usual Euclidian distance metric

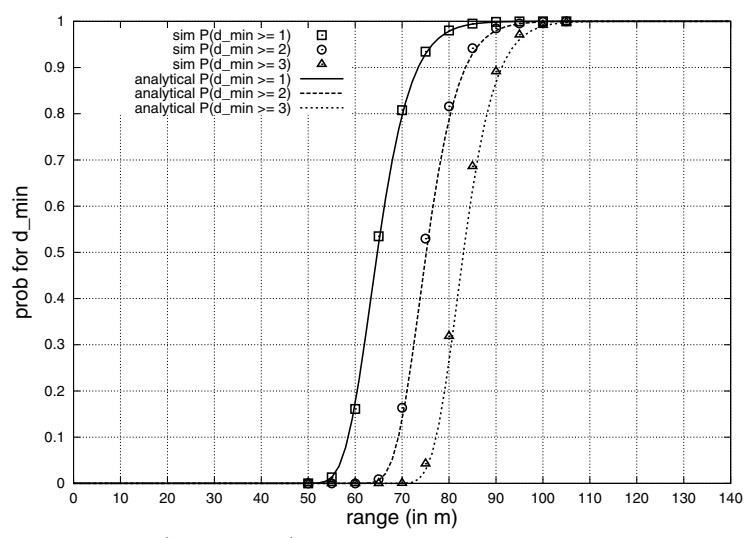

b. $P\left(d_{\min } \geq n_{0}\right)$ with toroidal distance metric

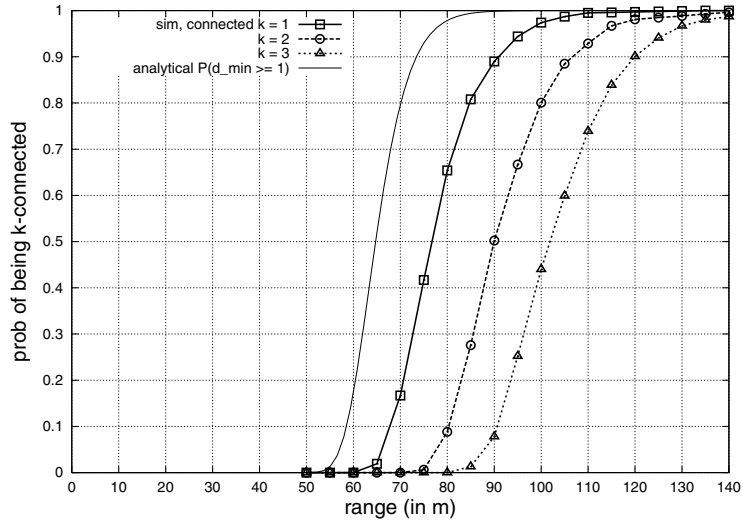

c. $P(k$-connected $)$ with usual Euclidian distance metric

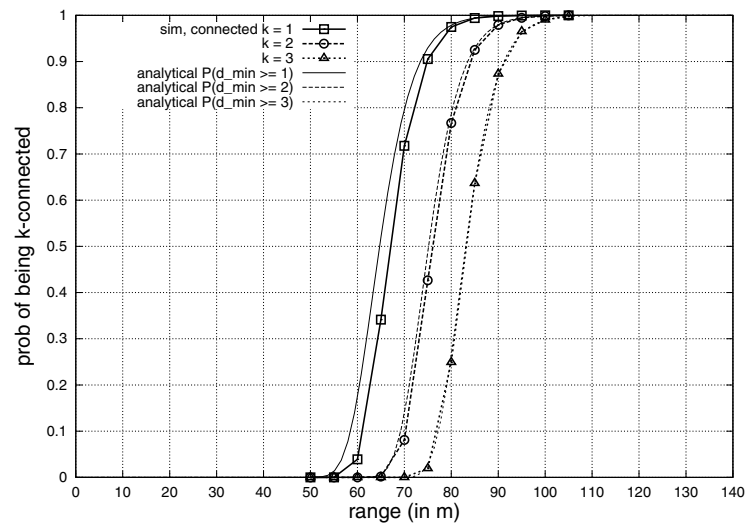

d. $P(k$-connected $)$ with toroidal distance metric

Figure 5: Probability that each node has at least $n_{0}$ neighbors, $P\left(d_{\min } \geq n_{0}\right)$; and prob. that the network is $k$-connected, $P(k$-connected $)$, both over $r_{0}$. Simulation for 500 nodes on $1000 \times 1000 \mathbf{m}^{2}\left(\rho=5 \cdot 10^{-4} \mathbf{m}^{-2}\right)$. The usual Euclidian distance causes a border effect whereas a toroidal distance metric avoids this effect.

first avoidance method is to divide the entire simulation area into two disjunct zones (as in [18]): a border zone $A_{b}$ with a width of at least $r_{0}$ and an inner zone $A_{\text {eff }}$. Only nodes that are located in the inner zone are considered for the statistics of the simulations. In other words, links connecting an inner node with a border node are only counted for the inner node. A disadvantage of this method is that the number of nodes that contribute to the statistics of the simulation decreases with increasing $r_{0}$. For example, a square simulation area of size $A=x_{\max }^{2}$ yields $A_{\text {eff }}=\left(x_{\max }-2 r_{0}\right)^{2}, r_{0}<x_{\max } / 2$. This results in a lower percentage of relevant nodes remaining for evaluation, $n_{e f f}=n \frac{A_{e f f}}{A}=n\left(1-\frac{2 r_{0}}{x_{\max }}\right)^{2}$, and therefore increases the required simulation time for confident results.

A second approach to avoid this border effect is to model the network topology in a way that nodes at the border are considered as being close to nodes at the opposite border and they are allowed to have links. Nodes at the right border can have links to nodes at the left border, and, analogously, nodes at the top can have links to nodes at the bottom. In other words, we use a toroidal distance metric - the flat simulation area becomes a torus [14]. Now, each node has the same expected node degree. This method is used in the following to compare the analytical curves with simulations.
Appendix B shows how the toroidal distance between two nodes in the simulation area is calculated. Its value is always smaller than or equal to the usual Euclidian distance.

Using toroidal distances between nodes, we repeated our simulation. Figure 5b shows the results in comparison to the analytical plots. Both curves match almost exactly, which confirms Theorem 1 and 2 .

\section{CONNECTIVITY}

We now go one step further and ask the question: What is the minimum transmission range $r_{0}$ such that, for given $\rho$, the network is connected? The connectivity of a wireless multihop network is one of its essential characteristics. It is often desired to achieve a connected network - both in a real network implementation and in a simulation. For example, several performance evaluations of routing protocols for ad hoc networks assume a connected topology (see, e.g., [19]).

The event $d_{\min }>0$ is a necessary (but not sufficient) condition for a graph to be connected. Thus, the value for a transmission range $r_{0}$ calculated with Equation (9) represents a lower bound for the radio transmission range that is needed to achieve a connected network. The same statement can be made for networks with a certain minimum 
node degree, i.e.,

$$
P(G \text { is } k \text {-connected }) \leq P\left(d_{\text {min }} \geq k\right)
$$

In other words, the implication ( $G$ is $k$-connected) $\Rightarrow$ $\left(d_{\min } \geq k\right)$ holds, but the reverse in not necessarily true. However, we can derive a useful relation for the disconnectivity of a network. Since a network with at least one isolated node is always disconnected, i.e., $\left(d_{\min }=0\right) \Rightarrow(G$ is disconnected), we can write

$$
P(G \text { is disconnected }) \geq 1-P\left(d_{\min }>0\right) .
$$

Example 1 (continued): If we set $r_{0}<55 \mathrm{~m}$ in our example scenario, the network will almost surely have an isolated node (see Fig. 5b). It will therefore almost surely be disconnected. Concerning connectivity, all we can say is that we need at least $r_{0}=83 \mathrm{~m}$ to achieve a connected network, since this range results almost surely in a network with no isolated node. However, we do not know how high we must set $r_{0}$ to achieve almost surely a connected network.

In practice, the upper bound for $P(G$ is $k$-connected $)$ does not help much if we do not know how tight it is. (Actually, a lower bound would be better.) In the following we show that this upper bound is a very tight bound, in particular for high probability values (which is certainly most interesting). As a result, we can conclude that Equation (17) can be used to calculate the range (or nodes density) that is needed to achieve an almost surely $k$-connected network.

\subsection{Analytical Derivation}

The key issue to obtain this result is to represent the topology of an ad hoc network as an undirected geometric random graph, denoted as $G(n, r)$. Let us first explain what a random graph, a geometric graph, and a geometric random graph is.

Definition 1: A random graph $G(n, p)$ is a graph with $n$ nodes in which each possible link is independently included with probability $p$.

The theory of random graphs is one the youngest research branches in graph theory. It uses probabilistic methods to solve problems in graph theory. Questions such as "what properties do 'most' graphs in various families have?" can be answered. An introduction to random graphs can be found in [13]; the books [20][21] cover their theory in detail.

The idea of modeling an ad hoc network as a random graph was already discussed in [22]. However, as said in that paper, the main obstacle in applying random graph theory for ad hoc networks is that random graphs do not allow taking into account correlations between different links. For example, if two ad hoc nodes $u$ and $v$ are physically very close to each other, and another ad hoc node $w$ is farther away, the existence of the links $(u, w)$ and $(v, w)$ is expected to be correlated in reality. Conventional random graph theory cannot model this correlation. Thus, results on the connectivity of random graphs, as presented in [20] (Chapter VII), cannot be used for our problem. However, there also exists the theory of geometric random graphs. The link between this special kind of random graphs and ad hoc networks has not been considered in [22].

Definition 2: Let the set of nodes $V=\{1, \ldots, n\}$ be a set of points in a metric space and let $\|\cdot\|$ denote an arbitrary distance norm in this space. A geometric graph $G(V, r)$ with radius $r$ is a graph with node set $V$ and the edge set $E=$ $\{u v \mid(u v \in V) \wedge(0<\|u-v\| \leq r\})$. In words, an edge is connecting each pair of nodes that are separated by a distance of at most $r$. Typically, in two dimensions, $V$ contains points in the unit square $[0,1]^{2}$ or unit disc, and $0<r<1$ (see, e.g., [23]). The distance norm can be e.g. $l_{2}$ (the Euclidian distance), $l_{1}$ (Manhattan distance), or $l_{\infty}$ (chessboard distance).

We are now able to give two definitions of what we mean by a geometric random graph (similar to $[23,24]$ ).

Definition 3: A random geometric graph $G(n, r)$ is a geometric graph in which the $n$ nodes are independently and uniformly randomly distributed in a metric space. In other words, it is a random graph in which the link existence probability $p$ between two nodes $u$ and $v$ is determined by their geometric distance in a way that $p=1$ for $\|u-v\| \leq r$ and $p=0$ otherwise.

If we use the standard Euclidian distance $l_{2}$ in twodimensions, it is obvious that the topology of a homogeneous ad hoc network can be represented as a random geometric graph $G(n, r)$ with $r=r_{0}$.

Proposition 1: A homogeneous ad hoc network with $n$ nodes, each with transmission range $r_{0}$, can be modeled as a geometric random graph $G\left(n, r_{0}\right)$.

Now, we apply a result on the property of geometric random graphs that was recently published in [24]. In this article, Penrose proved that if $n$ is high enough, then with high probability, if one starts with an empty graph (i.e., only isolated nodes) and adds the corresponding links as $r_{0}$ increases, the resulting graph becomes $k$-connected at the moment it achieves a minimum degree $d_{m i n}$ of $k$. So, for $k=1$, the network becomes connected at the moment where $r_{0}$ is large enough to achieve $d_{\min }(G)>0$ with high probability. In other words,

$$
P(G \text { is } k \text {-connected })=P\left(d_{\min } \geq k\right)
$$

for $P\left(d_{\min } \geq k\right)$ almost one. In fact, Penrose's theorem was proven to be true for any $l_{p}$ metric in any dimension higher than one, but it is not valid in one dimension [24].

This property of geometric random graphs is analogous to a property of conventional (non-geometric) random graphs: If links are added to an empty graph in an order chosen uniformly from the $\left(\begin{array}{l}n \\ 2\end{array}\right)$ ! possible node pairs, then with high probability, for large $n$ the resulting graph becomes $k$ connected at the instant when it achieves a minimum degree of $k$ (see [13]).

\subsection{Simulations and Discussion}

Let us now investigate the connectivity behavior of homogeneous ad hoc networks by simulation and therefore prove our analytical result. We repeat the experiment of Section 4 and now measure for each of the 100000 random topologies the connectivity of the network $(k=1,2,3)$. The complexity of our tests for connectedness and 2-connectedness is $O(n+m)$, the complexity of the test for 3-connectedness is $O(n(n+m))$. Most of the following results required extensive simulation time.

Figures $5 \mathrm{c}$ and $\mathrm{d}$ show the simulation results for $n=500$ nodes on a simulation area $A=1000 \times 1000 \mathrm{~m}^{2}$ using a usual distance metric and a toroidal distance metric, respectively. Applying the usual distance metric to link the nodes again causes a border effect, since nodes at the border are isolated with a higher probability and therefore the network gets disconnected. The simulation with the toroidal distance shows the desired result. For almost surely con- 


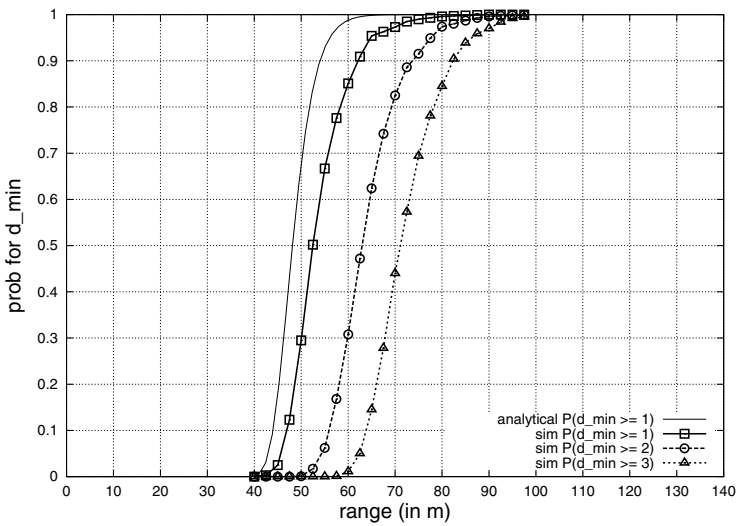

a. $P\left(d_{\min } \geq n_{0}\right)$ with usual Euclidian distance metric

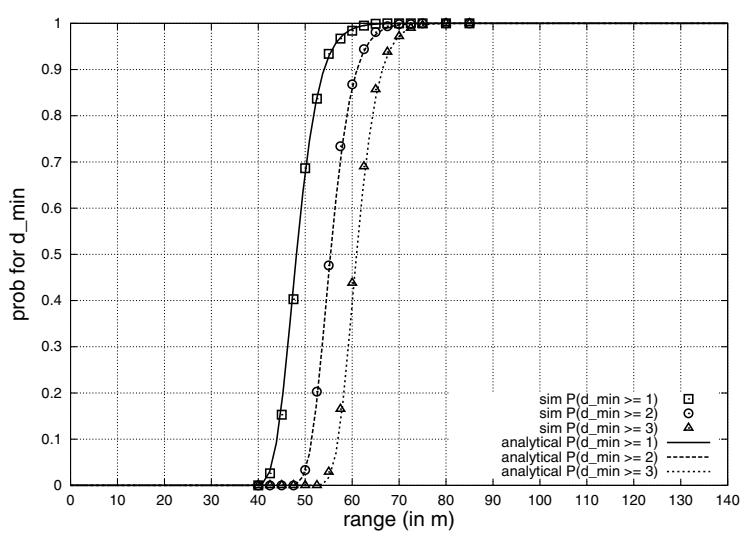

b. $P\left(d_{\min } \geq n_{0}\right)$ with toroidal distance metric

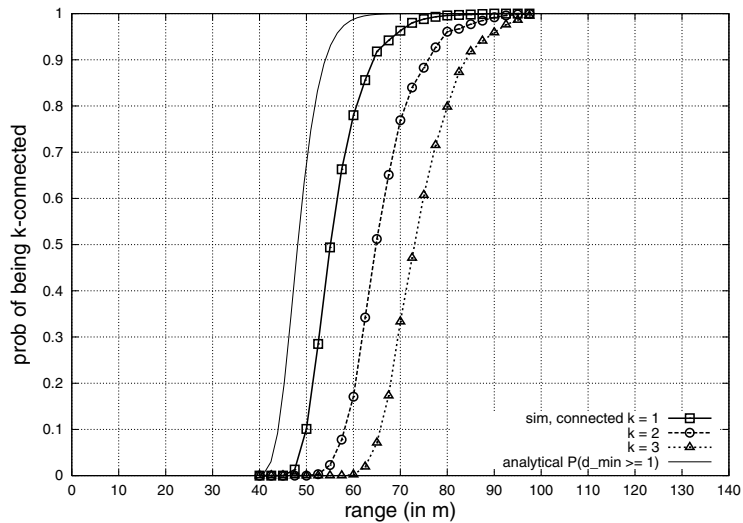

c. $P(k$-connected $)$ with usual Euclidian distance metric

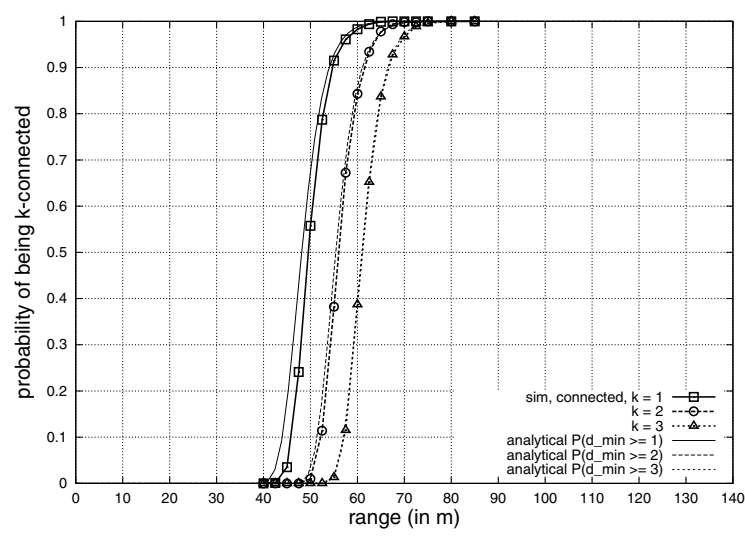

d. $P(k$-connected $)$ with toroidal distance metric

Figure 6: Simulation results for $n=1000$ nodes uniformly distributed on $A=1000 \times 1000 \mathbf{m}^{2}\left(\rho=10^{-3} \mathbf{m}^{-2}\right)$

nected networks, i.e., $P(G$ is $k$-connected $) \rightarrow 1$, the property $P(G$ is $k$-connected $)=P\left(d_{\text {min }} \geq k\right)$ holds (compare Fig. $5 \mathrm{~b}$ and $\mathrm{d})$.

Example 1 (continued): To achieve an almost surely connected network, the same range is required as to achieve a network with no isolated node $\left(r_{0}>83 \mathrm{~m}\right.$ for toroidal distances). The range must be increased to $r_{0} \approx 100 \mathrm{~m}$ to obtain a 3-connected network.

Also for lower connectivity probabilities, say between 0.8 and 1 , the curve for $P\left(d_{\min } \neq 0\right)$ serves as a good approximation for $P(G$ is connected $)$. This is especially true for $k \geq 2$. For example, $P(G$ is 3 -connected $) \approx P\left(d_{\min } \geq 3\right)$ holds for all probability values not just for $P\left(d_{\min } \geq k\right) \rightarrow 1$.

Interestingly, the comparison of Fig. 5a and c shows us that Equation (20) also seems to be valid in a bounded simulation area with border effects. In practice this means that it is sufficient to find out the critical range (or density) that almost surely creates a network with $d_{\min } \geq k$. This critical value is then also the critical value for $k$-connectivity. However, in this case, the curves show a significant difference for probabilities below $0.95 \%$.

Another interesting observation is that the probability of being $k$-connected changes rather fast from 0 to 1 as $r_{0}$ increases. Whereas a network with $n=500$ nodes on $10^{6} \mathrm{~m}^{2}$ is very unlikely to be connected for $r_{0}=60 \mathrm{~m}$, a little higher range $\left(r_{0}=75 \mathrm{~m}\right)$ yields an almost surely connected network. This observation is in accordance with the so-called "phase transition" phenomenon in random graph theory (see [13], Chapter VII, and [25]). It says that most standard properties of random graphs arise rather suddenly. The same observation can be made if we increase $\rho$ for a given $r_{0}$ (see Figs. 3 and 4 ).

Additional simulation results for $n=1000$ on $1000 \times$ $1000 \mathrm{~m}^{2}$ (Fig. 6), $n=2000$ on $2000 \times 2000 \mathrm{~m}^{2}$ (Fig. 7), and $n=100$ on $800 \times 800 \mathrm{~m}^{2}$ (Fig. 8), and $r_{0}=20 \mathrm{~m}$ on $500 \times 500 \mathrm{~m}^{2}$ (see Fig. 9) underline all these observations, and we can state the following theorem.

Theorem 3 (The probability for $k$-connectivity of a homogeneous ad hoc network): The probability that an ad hoc network with $n \gg 1$ nodes, each node with a transmission range $r_{0}$, and a homogeneous node density $\rho$ is $k$-connected is

$$
P(G \text { is } k \text {-connected }) \approx P\left(d_{\min } \geq k\right)
$$

for $P\left(d_{\min } \geq k\right)$ almost one. If border effects are eliminated, we can calculate this probability as

$$
\begin{gathered}
P(G \text { is } k \text {-connected }) \approx P\left(d_{\min } \geq k\right)= \\
=\left(1-\sum_{N=0}^{k-1} \frac{\left(\rho \pi r_{0}^{2}\right)^{N}}{N !} \cdot e^{-\rho \pi r_{0}^{2}}\right)^{n}
\end{gathered}
$$

for high probabilities $P\left(d_{\min } \geq k\right)$. From this equation, the threshold value for the transmission range $r_{0}$ (or the node density $\rho=n / A$ ) required to achieve an almost surely $k-$ 


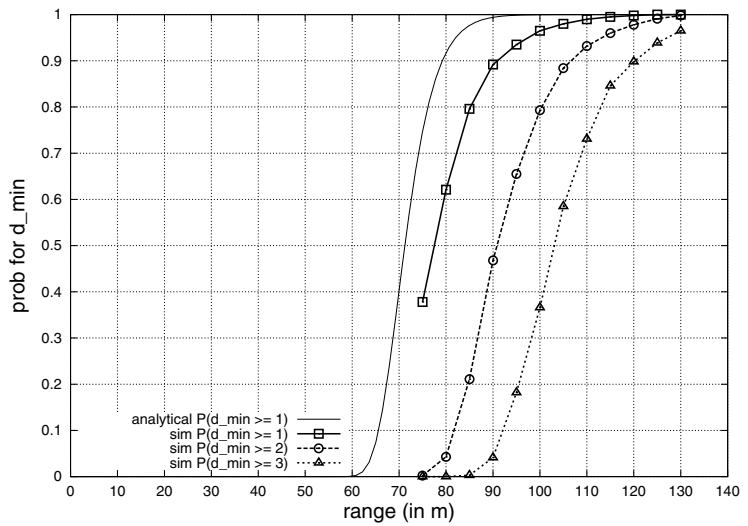

a. $P\left(d_{\min } \geq n_{0}\right)$ with usual Euclidian distance metric

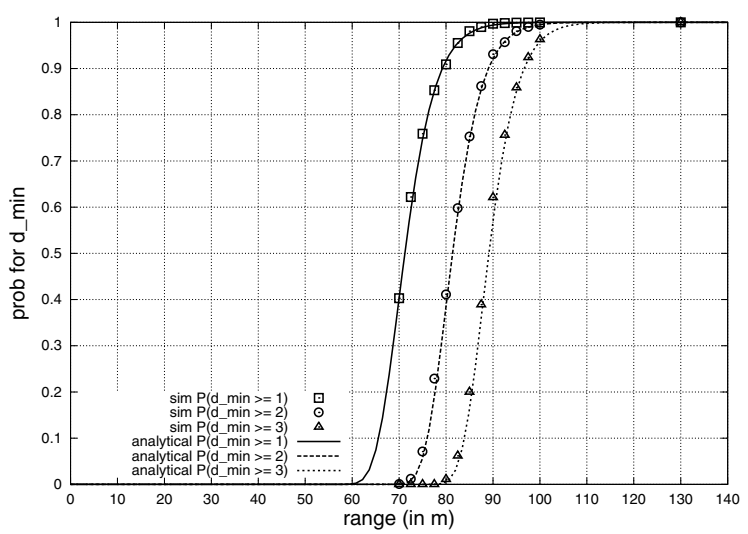

b. $P\left(d_{\min } \geq n_{0}\right)$ with toroidal distance metric

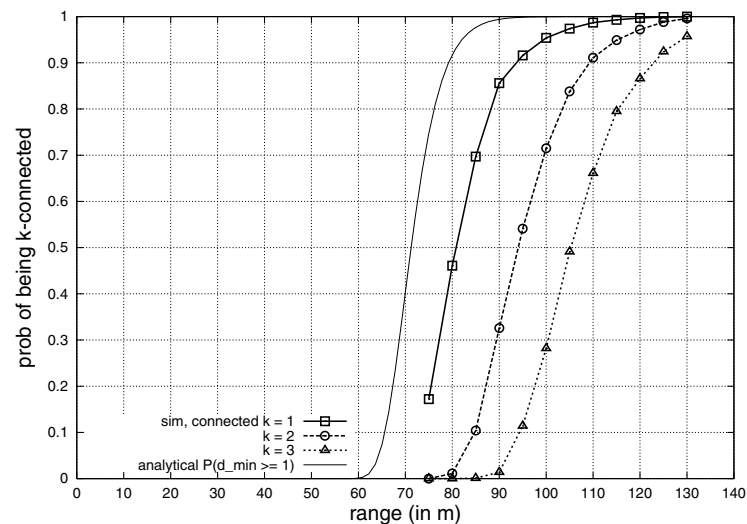

c. $P(k$-connected $)$ with usual Euclidian distance metric

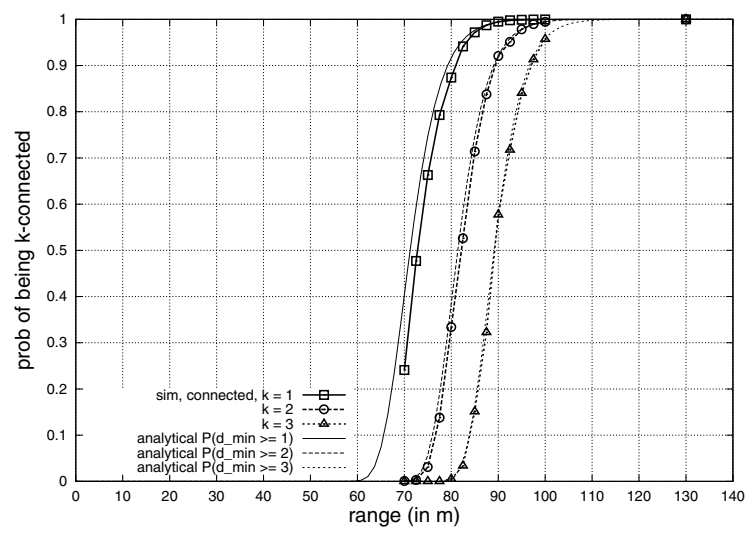

d. $P(k$-connected $)$ with toroidal distance metric

Figure 7: Simulation results for $n=2000$ nodes uniformly distributed on $A=2000 \times 2000 \mathbf{m}^{2}\left(\rho=5 \cdot 10^{-4} \mathbf{m}^{-2}\right)$

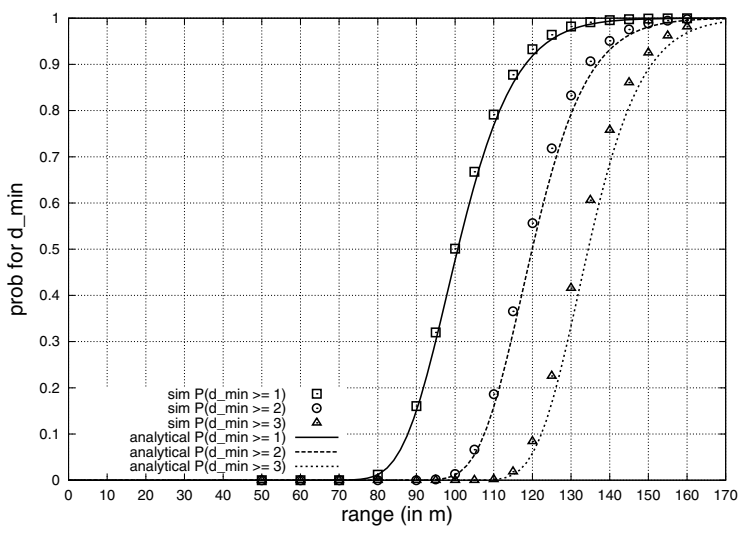

a. $P\left(d_{\min } \geq n_{0}\right)$ with toroidal distance metric

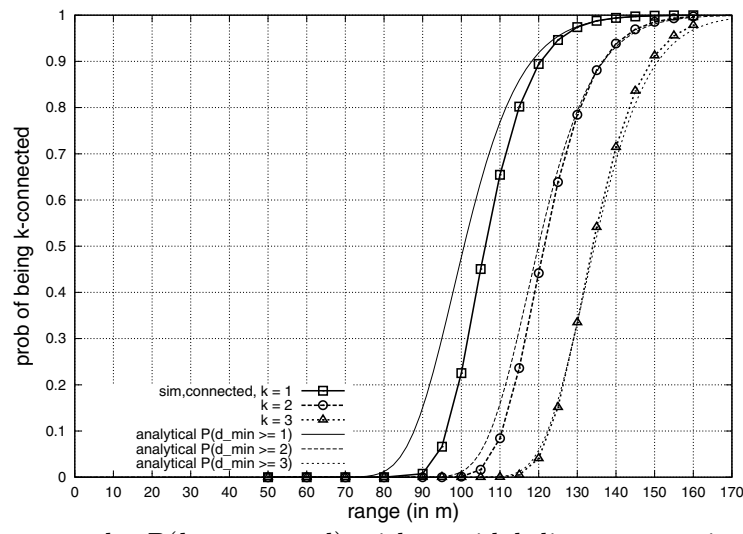

b. $P(k$-connected $)$ with toroidal distance metric

Figure 8: Simulation results for $n=100$ nodes uniformly distributed on $A=800 \times 800 \mathbf{m}^{2}\left(\rho=1.5625 \cdot 10^{-4} \mathbf{m}^{-2}\right)$

connected network can be determined. For example, the 99\%-contour lines in Figures 3 and 4 can be used to find out critical $\left(n, r_{0}\right)$-pairs.

In a bounded area with border effects, Equation (22) serves as an upper bound for $P(G$ is $k$-connected $)$. In other words, the threshold range (or density) that is required to achieve a certain connectivity is always higher as in the case without border effects.

Example 2 (Design of a large-scale wireless sensor network): A wireless sensor network should cover an area of size $A=500 \times 500 \mathrm{~m}^{2}$. Since all sensors exchange information, e.g., for environmental monitoring, the network should be connected. The sensors are equipped with transceivers that transmit a range of $r_{0}=20 \mathrm{~m}$ in free space and do not perform power control. How many sensors do we need to dis- 


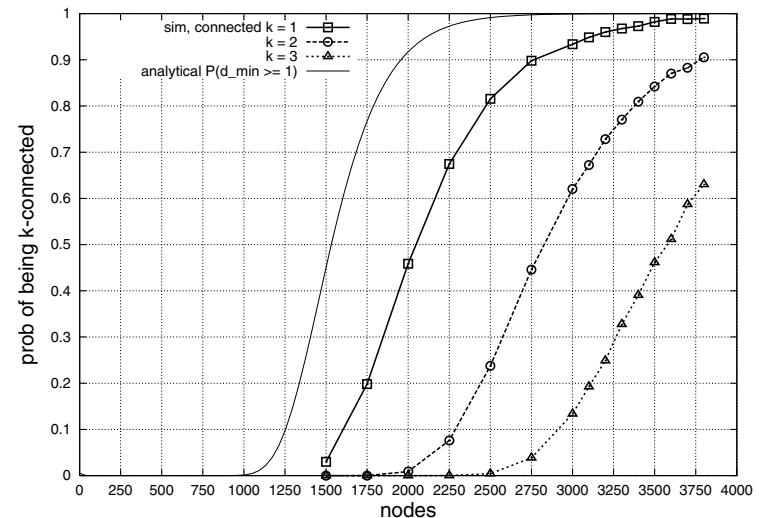

Figure 9: Simulation results for $n$ nodes with $r_{0}=$ $20 \mathbf{m}$ uniformly distributed on $A=500 \times 500 \mathbf{m}^{2}, P(k-$ connected), usual Euclidian distance, 3000 random topologies (see Example 2)

tribute over the area? With (8) and (19), we can state that a network with less than 1200 sensors on this area is almost surely disconnected, and according to (22), we need about 2500 sensors to obtain with high probability a connected network. Since the border effects of the real network implementation decrease the connectivity, we need even more sensors. A simulation with a usual Euclidean distance metric (see Fig. 9) shows that $n=2500$ creates a connected network with a probability of $p=82.5 \%$. If we distribute 3250 sensors, we will almost surely obtain a connected network $(p>95 \%)$.

\section{IMPACT OF MOBILITY}

Our results on connectivity are also applicable in long-run simulations with mobile nodes, provided that the network consists of many nodes $n \gg 1$ that are uniformly randomly distributed over an area $A \gg r_{0}^{2} \pi$ at each time step. More specifically, the used random mobility model must result in a uniform node distribution, all nodes move randomly and independently of each other, and their movement is not bounded to a certain subarea. Let us discuss the applicability of two common random mobility models in more detail (see also [26]).

A very popular and frequently used mobility model in ad hoc networking research is the random waypoint model (see, e.g., [27, 28, 29, 30]). It is a straightforward stochastic model that describes the movement behavior of a mobile network node in a two-dimensional system area as follows: A node randomly chooses a destination point in the area and moves with constant speed to this point. After waiting a certain pause time, it chooses a new destination, moves to this destination, and so on. As shown in Fig. 10, this mobility model does not result in a uniform node distribution. The reason for this behavior is obvious: Nodes located at the edges or borders of the simulation area are very likely to move back toward the middle of the area. For example, a node located at $(x, y)=(100 \mathrm{~m}, 100 \mathrm{~m})$ on a $1000 \times 1000 \mathrm{~m}^{2}$ area chooses with much higher probability a new destination point in the direction $\varphi \in] 0, \frac{\pi}{2}$ [ than a point toward a border or the edge. Most likely, it chooses a destination point that requires the node to pass the middle of the simulation area; in this example, $\varphi=\frac{\pi}{4}$. Such a non-uniform node

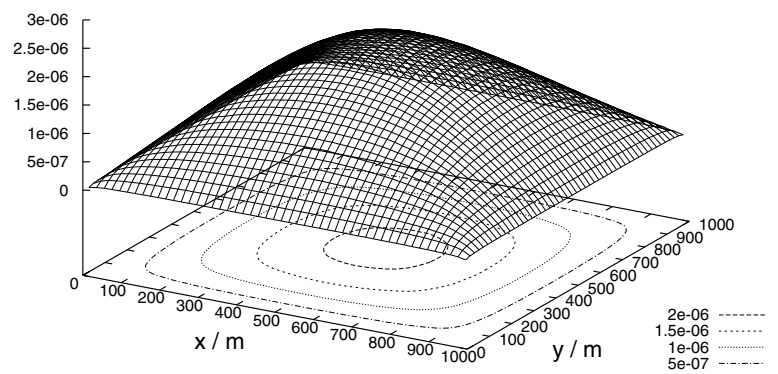

a. Square simulation area

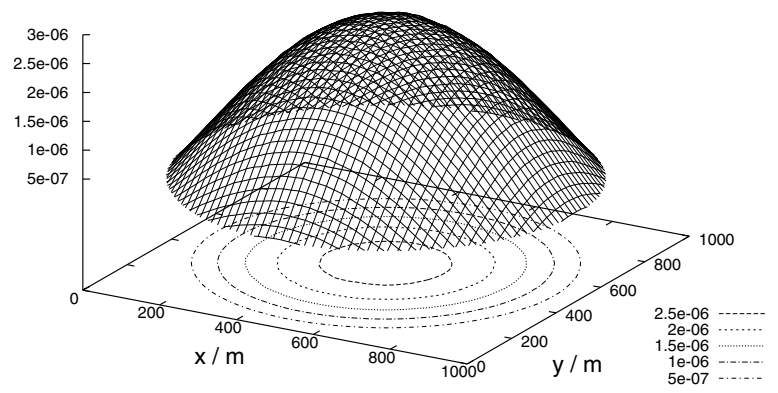

b. Circular simulation area (disc)

Figure 10: Spatial node distribution resulting from the random waypoint mobility model (sim. results)

distribution makes it much more difficult to derive analytical connectivity results. Even if a toroidal distance metric is used, the nodes at the border will on average have less neighbors than nodes in the middle of the area.

An alternative to the random waypoint model is a simple random direction model (see, e.g., [26, 31]) in which a new direction $\varphi \in[0 \ldots 2 \pi[$, rather than a destination point, is chosen after a random time. The duration of these movement epochs follow, for example, a negative exponential distribution. This model has approximately the same complexity and programming effort as the random waypoint model. Nodes always have a uniformly distributed angle within $[0 \ldots 2 \pi[$. They can also cross the borders of the simulation area and should then be bounced back or "wrapped around" to the other side of the area, which results in a uniform node distribution. Such a model should be preferred, if a homogeneous distribution is desired.

If all assumptions are met, the probability values of a static scenario can be interpreted as time in a mobile scenario: For a total simulation time $T$, the network is expected to have a minimum degree $d_{\min } \geq k$ during a time $P\left(d_{\min } \geq k\right) \cdot T$.

Example 1 (continued): We would like to investigate a distributed algorithm in a mobile ad hoc network and require that the network should be almost surely connected. The $n=500$ nodes move according to a random direction model and "bounce back" when they reach the border of the $A=$ $1000 \times 1000 \mathrm{~m}^{2}$ system area. We choose $r_{0} \geq 83 \mathrm{~m}$ to be sure that the network is connected for $99 \%$ of the simulation time (toroidal distance metric). If we do not use a toroidal distance metric, we can choose $r_{0} \geq 107 \mathrm{~m}$ in order to obtain the same confidentiality (see Fig. $5 \mathrm{c}$ ).

Note that these statements can be made for the given network topology model of Section 2. If we use a more 
detailed radio propagation model, which considers effects like Doppler shift, the connectivity will be influenced by the mobility behavior of the nodes.

\section{RELATED WORK}

Before we conclude, let us compare our results with related papers. Cheng and Robertazzi [32] studied the percolation of a node's broadcast in a multihop network. In particular, they examined the influence of $\rho$ and $r_{0}$ on the extend of the broadcast propagation. Philips et al. [33] discussed how the expected number of neighbors of a node must grow with the system area to maintain connectivity. This problem was resumed by Piret in [34] for one dimension. Recently, Gupta and Kumar [35] have shown that if the radio transmission range of $n$ nodes in a disc of unit area is set to $r_{0}=\sqrt{\frac{\log n+c(n)}{n \pi^{2}}}$, the resulting wireless multihop network is asymptotically connected with probability one if and only if $c(n) \rightarrow \infty$. They also obtained a necessary and a sufficient condition on $r_{0}$ for connectivity. Also Santi, Blough, and Vainstein [11] analyzed the radio range assignment problem. Among others, they derived bounds for the probability that a node is isolated - denoted as $P(d=0)$ in our paper. Furthermore, they stated a lower bound for $P$ (connected) for nodes on a one-dimensional (bounded) line $\left[0, x_{\max }\right]$ :

$$
P(\text { connected }) \geq 1-\left(x_{\max }-r_{0}\right)\left(1-\frac{r_{0}}{x_{\max }}\right)^{n}
$$

Two dimensional system areas have been considered by simulation.

Our paper employed geometric random graph theory and various simulations to find the link between a multihop network with isolated nodes and a connected topology. One of our contributions is a convenient expression that enables the determination of $\left(r_{0}, n\right)$ pairs that result in an almost surely connected topology for a given area $A$. Moreover, all related articles consider only 1 -connectivity and isolated nodes; none of them investigated $k$-connectivity $(k>1)$ and $d_{\min }>1$, which has been treated in detail in this paper. Also the impact of mobility has not been considered before.

Another related article is written by Appel and Russo [36]. They perform a mathematical study of the asymptotic minimum node degree $d_{\min }$ of a graph on uniform points in $d$ dimensions for $n \rightarrow \infty$. Chessboard distances between nodes (the $l_{\infty}$ metric) are used to form the graph. In the case of wireless networks, we must consider Euclidian distances and also finite values of $n$.

\section{CONCLUSIONS}

This paper investigated the connectivity of a wireless multihop network with homogeneous random node distribution. Among other things, we modeled the network as a geometric random graph and derived an analytical expression that allows the determination of the required transmission range $r_{0}$ that creates, for a given node density $\rho$, an almost surely $k$-connected network. Equivalently, if the maximum range $r_{0}$ of the nodes is given (e.g., say $r_{0}=250 \mathrm{~m}$ for wireless LANs), we can find out how many nodes of this type are needed to cover a certain area with a $k$-connected network. These problems have also been investigated by simulation in various scenarios, with and without border effects.
Our results are of practical value for researchers and developers who perform simulations or design of static and mobile ad hoc networks. For example, good simulation parameters can be chosen to achieve a connected mobile ad hoc network during most of the simulation time. Our results can also be used in the implementation of real ad hoc systems, in particular, if topology control does not find application, because low-cost nodes are used or it produces too much signaling overhead and processing load in the nodes. If topology control methods are used, the calculated critical radio range $r_{0}$ could serve as a starting value. Finally, we would like to note that the results of this paper are applicable to any kind of multihop system (not only radio networks, but also, e.g., multihop underwater acoustic networks [37]).

\section{Acknowledgement}

The author thanks Alejandro C. Frery (UFPE, Brazil) for a helpful conversation on point processes.

\section{REFERENCES}

[1] B. M. Leiner, D. L. Nielson, and F. A. Tobagi, eds., Proceedings of the IEEE, Special issue on Packet Radio Networks, vol. 75, Jan. 1987.

[2] Z. J. Haas, M. Gerla, D. B. Johnson, C. E. Perkins, M. B. Pursley, M. Steenstrup, and C.-K. Toh, eds., IEEE Journal on Sel. Areas in Comm., Special issue on Wireless Ad Hoc Networks, vol. 17, Aug. 1999.

[3] P. Kermani and N. H. Vaidya, eds., IEEE Personal Comm., Special issue on Advances in Mobile Ad Hoc Networking, Feb. 2001.

[4] C. E. Perkins, ed., Ad Hoc Networking. Addison-Wesley, 2001.

[5] G. J. Pottie and W. J. Kaiser, "Wireless integrated network sensors," Comm. of the ACM, May 2000.

[6] Y.-D. Lin and Y.-C. Hsu, "Multihop cellular: A new architecture for wireless communications," in Proc. IEEE Infocom, (Tel Aviv, Israel), Mar. 2000.

[7] W. Kellerer, C. Bettstetter, C. Schwingenschlögl, P. Sties, K.-E. Steinberg, and H.-J. Vögel, "(Auto)Mobile communication in a heterogeneous and converged world," IEEE Pers. Comm., Dec. 2001.

[8] H. Hartenstein, B. Bochow, A. Ebner, M. Lott, M. Radimirsch, and D. Vollmer, "Position-aware ad hoc wireless networks for inter-vehicle communications: The Fleetnet project," in Proc. ACM Intern. Symp. on Mobile Ad Hoc Netw. and Comp. (MobiHoc), (Long Beach, CA), Oct. 2001.

[9] R. Ramanathan and R. Rosales-Hain, "Topology control of multihop wireless networks using transmit power adjustment," in Proc. IEEE Infocom, (Tel Aviv, Israel), Mar. 2000.

[10] R. Wattenhofer, L. Li, P. Bahl, and Y.-M. Wang, "Distributed topology control for power efficient operation in multihop wireless ad hoc networks," in Proc. IEEE Infocom, (Anchorage, Alaska), Apr. 2001.

[11] P. Santi, D. M. Blough, and F. Vainstein, "A probabilistic analysis for the radio range assignment problem in ad hoc networks," in Proc. ACM Intern. Symp. on Mobile Ad Hoc Netw. and Comp. (MobiHoc), (Long Beach, CA), Oct. 2001.

[12] R. Diestel, Graph Theory. Springer, 2nd ed., 2000. 
[13] B. Bollobás, Modern Graph Theory. Springer, 1998.

[14] N. A. C. Cressie, Statistics for Spatial Data. John Wiley \& Sons, 1991.

[15] H. Takahi and L. Kleinrock, "Optimal transmission ranges for randomly distributed packet radio terminals," IEEE Trans. on Comm., Mar. 1984.

[16] A. Papoulis, Probability, random variables, and stochastic processes. McGraw-Hill, 1984.

[17] M. Matsumoto and T. Nishimura, "Mersenne twister: A 623-dimensionally equidistributed uniform pseudorandom number generator," ACM Trans. on Modeling and Computer Simulation, Jan. 1998.

[18] C. Bettstetter and O. Krause, "On border effects in modeling and simulation of wireless ad hoc networks," in Proc. IEEE Intern. Conf. on Mobile and Wireless Comm. Netw. (MWCN), (Recife, Brazil), Aug. 2001.

[19] M. K. Marina and S. R. Das, "On-demand multipath distance vector routing in ad hoc networks," in Proc. Intern. Conf. for Network Procotols (ICNP), (Riverside, CA), Nov. 2001.

[20] B. Bollobás, Random Graphs. Academic Press, 1985.

[21] S. Janson, A. Rucinski, T. Luczak, and T. Uczak, Theory of Random Graphs. Wiley, May 2000.

[22] I. Chlamtac and A. Faragó, "A new approach to the design and analysis of peer-to-peer mobile networks," ACM/Baltzer Wireless Networks, vol. 5, Aug. 1999.

[23] J. Díaz, M. D. Penrose, J. Petit, and M. Serna, "Convergence theorems for some layout measures on random lattice and random geometric graphs," Combinatorics, Probability, and Computing, no. 6 , pp. 489-511, 2000.

[24] M. D. Penrose, "On k-connectivity for a geometric random graph," Wiley Random Structures and Algorithms, vol. 15, no. 2, pp. 145-164, 1999.

[25] P. Erdös and A. Rényi, "On the evolution of random graphs," Publ. Math. Inst. Hungar. Acad. Sci., vol. 5, pp. $17-61,1960$.

[26] C. Bettstetter, "Mobility modeling in wireless networks: Categorization, smooth movement, and border effects," ACM Mobile Computing and Communications Review, vol. 5, no. 3, 2001.

[27] J. Broch, D. A. Maltz, D. B. Johnson, Y.-C. Hu, and J. Jetcheva, "A performance comparison of multi-hop wireless ad hoc network routing protocols," in Proc. ACM Intern. Conf. on Mobile Comp. and Netw. (MobiCom), (Dallas, TX), Oct. 1998.

[28] E. M. Royer and C. E. Perkins, "Multicast operation of the ad-hoc on-demand distance vector routing protocol," in Proc. ACM Intern. Conf. on Mobile Comp. and Netw. (MobiCom), (Seattle, WA), 1999.

[29] S. R. Das, C. E. Perkins, and E. M. Royer, "Performance comparison of two on-demand routing protocols for ad hoc networks," in Proc. IEEE Infocom, (Tel Aviv, Israel), 2000.

[30] G. Holland and N. H. Vaidya, "Analysis of TCP performance over mobile ad hoc networks," in Proc. ACM Intern. Conf. on Mobile Comp. and Netw. (MobiCom), (Seattle, WA), Aug. 1999.

[31] R. A. Guérin, "Channel occupancy time distribution in a cellular radio system," IEEE Trans. on Vehicular Technology, vol. 36, Aug. 1987.
[32] Y.-C. Cheng and T. G. Robertazzi, "Critical connectivity phenomena in multihop radio models," IEEE Trans. on Communications, vol. 37, July 1989.

[33] T. K. Philips, S. S. Panwar, and A. N. Tantawi, "Connectivity properties of a packet radio network model," IEEE Trans. on Inform. Theory, Sept. 1989.

[34] P. Piret, "On the connectivity of radio networks," IEEE Trans. on Information Theory, Sept. 1991.

[35] P. Gupta and P. R. Kumar, "Critical power for asymptotic connectivity in wireless networks," in Stochastic Analysis, Control, Optimization and Applications, pp. 547-566, Birkhäuser, 1998.

[36] M. J. B. Appel and R. P. Russo, "The minumum vertex degree of a graph on uniform points in $[0,1]^{d}$," Adv. in Applied Probability, vol. 29, pp. 582-594, 1997.

[37] J. G. Proakis, E. M. Sozer, J. A. Rice, and M. Stojanovic, "Shallow water acoustic networks," IEEE Personal Communications, Nov. 2001.

\section{APPENDIX}

\section{A. Expected Nearest Neighbor Distance}

The distance of a node to its closest neighboring node is denoted as its nearest neighbor distance $\xi$. For a homogeneous Poisson process in $R^{2}$, the probability density function of the nearest neighbor distance is $p(\xi)=2 \pi \rho \xi e^{-\rho \pi \xi^{2}}$ for $\xi>0$ (Section 4). The expected value for $\xi$ can be calculated as

$$
E(\xi)=\int_{0}^{\infty} \xi p(\xi) d \xi=2 \pi \rho \int_{0}^{\infty} \xi^{2} e^{-\rho \pi \xi^{2}} d \xi .
$$

Using the substitution $\rho \pi=a$ and the solution of the definite integral

$$
\int_{0}^{\infty} \xi^{b} e^{-a \xi^{2}} d \xi=\frac{\Gamma\left(\frac{b+1}{2}\right)}{2 a^{\left(\frac{b+1}{2}\right)}}
$$

with the gamma function $\Gamma(\cdot)$, we obtain

$$
E(\xi)=\frac{\Gamma\left(\frac{3}{2}\right)}{\sqrt{\rho \pi}}=\frac{\sqrt{\pi} / 2}{\sqrt{\rho \pi}}=\frac{1}{2 \sqrt{\rho}} .
$$

\section{B. Toroidal Distance}

A toroidal metric is used in this paper to eliminate border effects (see Section 4). Let $d\left(\left(\begin{array}{l}x_{1} \\ y_{1}\end{array}\right),\left(\begin{array}{l}x_{2} \\ y_{2}\end{array}\right)\right)$ denote the usual Euclidian distance between two points $\left(\begin{array}{l}x_{1} \\ y_{1}\end{array}\right)$ and $\left(\begin{array}{l}x_{2} \\ y_{2}\end{array}\right)$ on a limited area $\left[0, x_{\max }\right]\left[0, y_{\max }\right]$. The toroidal distance is then

$$
\begin{array}{r}
d_{T}\left(\left(\begin{array}{l}
x_{1} \\
y_{1}
\end{array}\right),\left(\begin{array}{l}
x_{2} \\
y_{2}
\end{array}\right)\right)=\min \left\{d\left(\left(\begin{array}{l}
x_{1} \\
y_{1}
\end{array}\right),\left(\begin{array}{l}
x_{2} \\
y_{2}
\end{array}\right)\right),\right. \\
d\left(\left(\begin{array}{c}
x_{1}+x_{\max } \\
y_{1}
\end{array}\right),\left(\begin{array}{l}
x_{2} \\
y_{2}
\end{array}\right)\right), d\left(\left(\begin{array}{c}
x_{1}-x_{\max } \\
y_{1}
\end{array}\right),\left(\begin{array}{l}
x_{2} \\
y_{2}
\end{array}\right)\right), \\
d\left(\left(\begin{array}{c}
x_{1} \\
y_{1}+y_{\max }
\end{array}\right),\left(\begin{array}{l}
x_{2} \\
y_{2}
\end{array}\right)\right), d\left(\left(\begin{array}{c}
x_{1} \\
y_{1}-y_{\max }
\end{array}\right),\left(\begin{array}{l}
x_{2} \\
y_{2}
\end{array}\right)\right), \\
d\left(\left(\begin{array}{l}
x_{1}+x_{\max } \\
y_{1}+y_{\max }
\end{array}\right),\left(\begin{array}{l}
x_{2} \\
y_{2}
\end{array}\right)\right), d\left(\left(\begin{array}{l}
x_{1}+x_{\max } \\
y_{1}-y_{\max }
\end{array}\right),\left(\begin{array}{l}
x_{2} \\
y_{2}
\end{array}\right)\right), \\
\left.d\left(\left(\begin{array}{l}
x_{1}-x_{\max } \\
y_{1}+y_{\max }
\end{array}\right),\left(\begin{array}{l}
x_{2} \\
y_{2}
\end{array}\right)\right), d\left(\left(\begin{array}{l}
x_{1}-x_{\max } \\
y_{1}-y_{\max }
\end{array}\right),\left(\begin{array}{l}
x_{2} \\
y_{2}
\end{array}\right)\right)\right\}
\end{array}
$$

where $d_{T}\left(\left(\begin{array}{l}x_{1} \\ y_{1}\end{array}\right),\left(\begin{array}{l}x_{2} \\ y_{2}\end{array}\right)\right) \leq d\left(\left(\begin{array}{l}x_{1} \\ y_{1}\end{array}\right),\left(\begin{array}{l}x_{2} \\ y_{1}\end{array}\right)\right)$. 